\title{
Simulation of Excess Nutrient Solution Volume of Greenhouse Cucumber Under Soilless Culture
}

\author{
Jing $\mathrm{Hu}^{1,2}$, Guopeng Chen ${ }^{1}$, Xinhao Gao ${ }^{1}$, Chunyun Zhang ${ }^{2}$, Yifang Wen ${ }^{1}$ \\ ${ }^{1}$ Suzhou Vocational Institute of Industrial Technology, Wuzhong, China \\ ${ }^{2}$ Anhui Science and Technology University, Bengbu, China \\ ${ }^{3}$ Key Laboratory of Modern Agriculture Equipment and Technology, Ministry of Education, Jiangsu \\ University, Zhenjiang, China.
}

Keywords: Excess nutrient solution; Absorption of substract; Root interception; Nutrient solution; Greenhouse cucumber.

\begin{abstract}
The prediction of excess nutrient solution volume was an essential part of re-used of nutrient solution system, and it was determined by volume of irrigation, substract, crop development stage and water content of substract. In order to found simulation model of excess nutrient solution volume, experiments were conducted at Venlo style greenhouse of jiangsu university on 4-11, 2013. Absorption of perlite and intercept of root of nutrient solution were studied, and a simulation model of excess nutrient solution was found. Other data from different experiment was used to validate the model. The result showed that the coefficients of determination between simulated and measured value based on the 1:1 line were 0.94 , and the root mean squared error between the simulated and the measured value was $5.8 \mathrm{ml}$ per plant. The model could give satisfactory prediction of excess nutrient solution volume. A simulation model of excess nutrient solution was firstly found and could afford theoretical guidance to design system of re-used nutrient solution.
\end{abstract}

\section{Introduction}

Soil-less cultivation has the following advantages compared with traditional soil cultivation mode: cheap, pollution-free and green food can be produced to meet people's life needs, and the efficiency of water fertilizer use can be improved [1-3]. Soil-less cultivation area is increased rapidly in China in recent years [4], soil-less cultivation area is increased rapidly to cause a series of problems [5], the most serious problem is increasing discharge of excess nutrient solution (namely the nutrient solution discharge liquid).

The nutrient solution recycling technology was frequently studied in the past. Current study focuses on the excess nutrient solution disinfection technology [5-7] and quick detection of nutrient content in excess nutrient solution [8]. The study on prediction of excess nutrient solution volume has not been reported till present. Currently, excess nutrient solution volume prediction relies mainly on the experience.

$\mathrm{Lu}$ Weiguang believes that greenhouse tomato excess nutrient solution volume should account for $40 \%$ of irrigation volume [9]. Song Weitang believes that the nutrient solution circulation is generally $40-60 \mathrm{~m} 3 / \mathrm{h}$, and the circulation quantity is $4-6$ times/d[6] for the cultivation system with the area of $667 \mathrm{~m} 2$. The simulation study on excess nutrient solution volume is not reported at present. Excess nutrient solution volume is related to crop growth stage, irrigation volume and substract absorption characteristics. Therefore, a prediction model of greenhouse cucumber excess nutrient solution volume is established through studying the relationship among substract absorption properties, crop growth stage and excess nutrient solution volume, and theoretical guidance is provided for recycling and utilization of excess nutrient solution. 


\section{Materials and Methods}

\subsection{Experiment Overview}

The experiment was carried out from March 2016 to November 2016 in Venlo experiment greenhouse of Jiangsu University. The experiment was divided into two parts, including experiment 2 for model construction and experiment 1 for model test. Experiment 1 was conducted from March 2016 to June 2016. The perlite load per barrel was respectively $200 \pm 10$ (T1), $500 \pm 10$ (T2) and 700 $\pm 10 \mathrm{~g}$ (T3). Plants were randomly grouped and arranged after each 30 plants were planned.

Experiment 2 was conducted from September 2016 to December 2016. The perlite dry weight each barrel was $500 \pm 10 \mathrm{~g}$. There were a total of 40 cultivation barrels for experiment cucumber, including 30 barrels with fixed plantation of cucumber and 10 barrels with unfixed plantation of cucumber (for calculation of root interception volume).

Excess nutrient solution collection device: the planted cucumber cultivation barrel was placed on the foam plastic board with slots on both sides. One end was slightly higher than the other end by $2 \mathrm{~cm}$. There was a excess nutrient solution collecting barrel on the lower end. Concrete conditions are shown in figure 1.

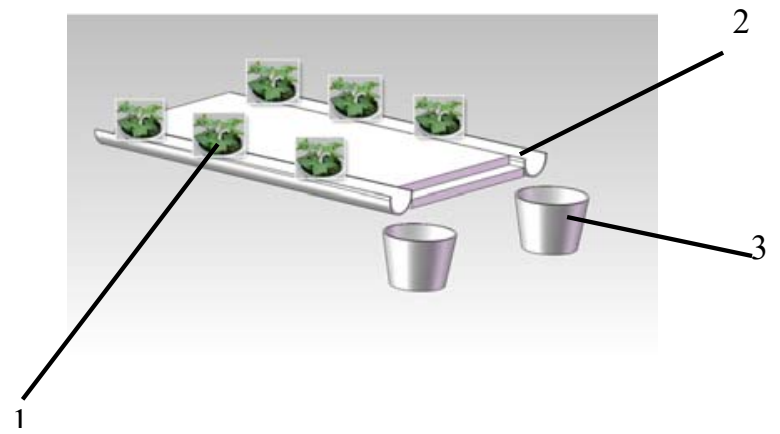

Figure 1. Excess nutrient solution recovery device (1. Cultivated crops; 2. Excess nutrient solution collecting tank; 3 . Excess nutrient solution collecting barrel)

\subsection{Measurement Items And Methods}

\subsubsection{Measurement of Excess Nutrient Solution Volume}

A measuring cylinder with precision of $0.1 \mathrm{ml}$ was used for excess nutrient solution volume in measuring excess nutrient solution collecting barrel after nutrient solution irrigation each time. The average value was adopted for obtaining excess nutrient solution volume per plant of greenhouse cucumber.

\subsubsection{Measurement of Matrix Water Content}

A rapid moisture tester (China, Wuhan Ruiling) was used for measuring water content of pearlite before nutrient solution irrigation each time. The measuring site was $10 \mathrm{~cm}$ from the base of cucumber plant, 5 plants were measured each time, and the average value is obtained.

\subsection{Model Description}

After nutrient solution is irrigated each time, a part of solution is absorbed by matrix, a part of solution exits in the matrix due to root interception. Excess nutrient solution is discharged. Therefore, it is assumed in the study that excess nutrient solution has no residue in excess nutrient solution collecting tank (one end is $2 \mathrm{~cm}$ higher) and the evaporation capacity is 0 ( cultivation barrel is covered with a plastic film), the excess nutrient solution volume is equal to nutrient solution irrigation volume after reduction of root interception volume and substract absorption volume. Concrete calculation method is shown as follows:

In the formula, $\mathrm{Y}$-excess nutrient solution volume $(\mathrm{ml})$

$$
\mathrm{Y}=\mathrm{N}-\mathrm{Sj}-\mathrm{Wr}
$$

$\mathrm{N}$-nutrient solution irrigation volume $(\mathrm{ml})$, and it is set by the greenhouse administrator.

Wr-root interception volume (ml)

$\mathrm{Sj}$-adsorbing capacity of matrix to nutrient solution (ml) 


\subsection{Calculation of Root Interception Volume}

Data of experiment 2 was utilized for calculation. Namely, the excess nutrient solution volume discharged from the barrel with crop cultivation was subtracted from the excess nutrient solution volume discharged from the barrel without crop cultivation. The difference value thereof is the interception of root to nutrient solution. The specific calculation is shown as follows:

In the formula, Wr-root interception volume (ml)

$$
\mathrm{Wr}=\mathrm{Y} 0-\mathrm{Yp}
$$

Y0-excess nutrient solution flow of cultivation barrel without crop cultivation (ml)

Yp-excess nutrient solution flow of cultivation barrel with crop cultivation (ml)

Root interception volume is closely related to root biomass, root biomass is increased with effective accumulative temperature rising. Data of experiment 2 is utilized to establish mathematical relationship between root interception volume and effective accumulative temperature (figure 2).

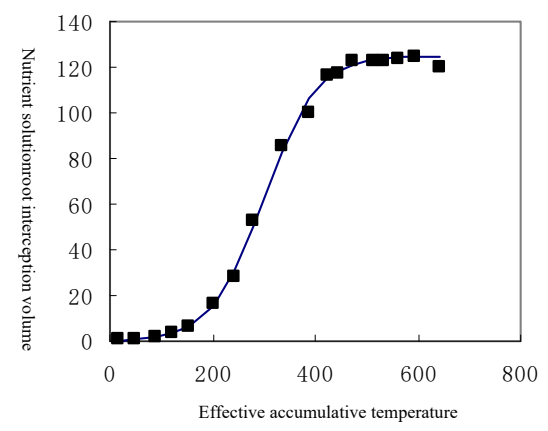

Figure 2. Relationship between effective accumulative temperature and nutrient solutionroot interception volume

The data of experiment 2 is used for establishing the relation formula between root interception volume and effective accumulative temperature (GDD) within the growth period of greenhouse cucumber.

Root interception volume can be calculated according to the following formula:

$$
\mathrm{Wr}(\mathrm{i})=\frac{125}{1+\mathrm{e}^{\frac{300-\mathrm{GDD}(\mathrm{i})}{50}}}
$$

In the formula, $\mathrm{Wr}$ (i) -root interception volume of the ith day in the growth period.

GDD (i) - effective accumulative temperature of the ith day in the growth period.

\subsection{Calculation of Substract Absorption Volume}

Substract absorption volume is equal to the result after subtracting current nutrient solution content in matrix from the maximum adsorbing capacity of matrix to nutrient solution. The following formula is adopted for calculation:

$$
\mathrm{Sj}=\mathrm{Sm}-\mathrm{Sb}
$$

In the formula: Sm- maximum adsorbing capacity of matrix (ml)

$\mathrm{Sb}$ - nutrient solution volume in matrix before nutrient solution irrigation $(\mathrm{ml})$

Wherein, Sm can be calculated through the following formula:

In the formula: M- matrix dry weight $(\mathrm{g})$;

$$
\mathrm{Sm}=\mathrm{a} * \mathrm{M}
$$

a- matrix adsorption exponent to nutrient solution $(\mathrm{ml} / \mathrm{g})$. In the study, perlite is adopted as cultivation matrix, and the adsorption exponent is $3.2493 \mathrm{ml} / \mathrm{g}$ according to experiment.

$\mathrm{Sb}$ can be calculated by adopting formula 7 :

$$
\mathrm{Sb}=\mathrm{a} * \mathrm{M} * \mathrm{~b}
$$

In the formula: $\mathrm{b}$ is matrix relative water content, and it can be obtained through near-infrared moisture meter.

\subsection{Calculation of Excess Nutrient Solution Volume}

Calculation method of substract absorption volume of the ith day is shown as follows:

$$
\mathrm{Sj}(\mathrm{i})=\mathrm{Sm}-\mathrm{Sb}(\mathrm{i})
$$




$$
\mathrm{Sb}(\mathrm{i})=\mathrm{a} * \mathrm{M} * \mathrm{~b}(\mathrm{i})
$$

Calculation method of excess nutrient solution volume of the ith day is shown as follows:

$$
\mathrm{Y}(\mathrm{i})=\mathrm{N}(\mathrm{i})-\mathrm{Sj}(\mathrm{i})-\mathrm{Wr}(\mathrm{i})
$$

\section{Results and Analysis}

The effective accumulative temperature of greenhouse cucumber during the whole growth period was obtained through the calculation method in the reference [10] according to the temperature data in the greenhouse. Formula (1) - (9) was adopted to calculate the excess nutrient solution analog quantity of the whole growth period opposite to the modeling data (experiment 1). It was compared with the measured value (figure 3). The results showed that the model has been consistency between simulated value and measured value of greenhouse cucumber single-plant excess nutrient solution volume. The determination coefficient R2 among model predicted value, measured value and 1:1 straight line was 0.94 . The root-mean-square error RMSE was $5.8 \mathrm{ml} /$ strain, thereby better simulating excess nutrient solution volume of the greenhouse cucumber.

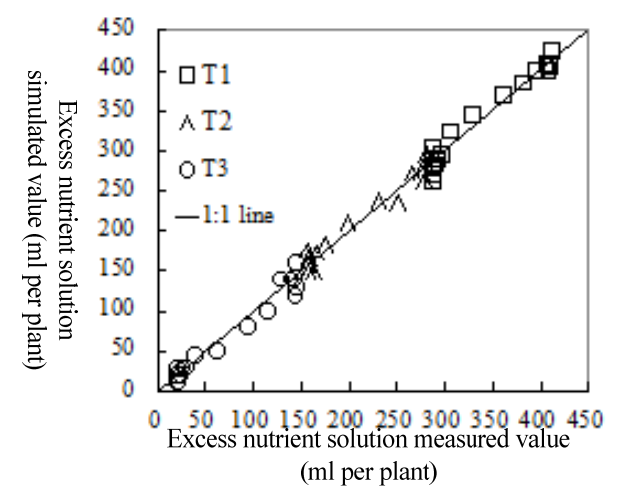

Figure 3. Comparison between excess nutrient solution volume simulated value and measured value

\section{Conclusion}

The daily excess nutrient solution volume in greenhouse production directly determines the maximum power of the nutrient solution recycling system. In the study, substract absorption volume and root interception volume are considered comprehensively for establishing the prediction model of greenhouse cucumber single-plant excess nutrient solution volume. The determination coefficient $\mathrm{R} 2$ between simulated value and measured value is 0.94 compared with the data of verification experiment. The root-mean-square error RMSE is $5.8 \mathrm{ml} / \mathrm{plant}$, thereby better predicting the single-plant excess nutrient solution volume of greenhouse cucumber.

\section{Acknowledgements}

This work is supported by Natural Science Major Basic Research Project of the Universities of Jiangsu Province (No.BK20161228), Science and Technology Plan Project of Suzhou (SYG201654), Anhui University Provincial Natural Science Research Projects (No. KJ2016A183) and Fund of Scientific Research Startup of Suzhou Vocational Institute of Industrial Technology.

\section{References}

[1]. Ziqin Wang, Dexin Gan, Yuelin Long. Advance in soilless culture researc. Agricultural Science \& Technology, 2013, 14(2): 269-278,323.

[2]. Liu Jing. Application and development of soil-less cultivation techniques. Northern Horticulture, 2012, 5 (16): 204-206. 
[3]. Han Yongfeng, Tu Yang, Wang Hongsheng. Overview and development countermeasure of soil-less cultivation. Hebei Journal of Forestry and Orchard Research, 2010, 25 (3): 296-298.

[4]. Yang Qichang, Wang Xiaoyun, Liu Wenke, etc. Study on new mode of soil-less cultivationnutrient solution cascade utilization. Chinese Agricultural Science Bulletin, 2006, 22 (7): 553-556.

[5]. Liu Wenke, Yang Qichang. Removal method of plant toxic substances in facility soil-less cultivation nutrient solution. Northern Horticulture, 2010 (16): 69-70.

[6]. Song Weitang, Wang Cheng, Hou Wenlong. Design and sterilization performance test of UV ozone combined nutrient solution disinfection machine. Transactions of the CSAE, 2011, 27 (2): $360-365$.

[7]. Song Weitang, Lu Shanshan. Research and application of slow filtration system in inhibiting the pathogens of soil transmission. Journal of China Agricultural University, 2009, 14 (1): 8-12.

[8]. Ruqin Fan, Xueming Yang, Hongtu Xie, et al. Determination of nutrient in hydroponic solutions using mid-infrared spectroscopy. Scientia Horticulturae, 2012, 144(6):48-54.

[9]. Lu Weiguang, Zhao Jingyin, Yao Zheng, etc. Investigation of nutrient solution management technique of modern greenhouse soil-less cultivation of tomato. Journal of Chinese vegetables, 2002 (1): 18-19, 28. 